This item was submitted to Loughborough's Research Repository by the author.

Items in Figshare are protected by copyright, with all rights reserved, unless otherwise indicated.

\title{
A two DoF finger for a biomechatronic artificial hand
}

PLEASE CITE THE PUBLISHED VERSION

http://content.iospress.com/articles/technology-and-health-care/thc00256

\section{PUBLISHER}

(C) IOS Press

\section{VERSION}

AM (Accepted Manuscript)

\section{PUBLISHER STATEMENT}

This work is made available according to the conditions of the Creative Commons Attribution-NonCommercialNoDerivatives 4.0 International (CC BY-NC-ND 4.0) licence. Full details of this licence are available at: https://creativecommons.org/licenses/by-nc-nd/4.0/

\section{LICENCE}

CC BY-NC-ND 4.0

\section{REPOSITORY RECORD}

Carrozza, M.C., B. Massa, Paolo Dario, Massimiliano Zecca, Silvestro Micera, and P. Pastacaldi. 2019. "A Two Dof Finger for a Biomechatronic Artificial Hand". figshare. https://hdl.handle.net/2134/17565. 


\section{A TWO DOF FINGER FOR A BIOMECHATRONIC}

\section{ARTIFICIAL HAND}

M.C. Carrozza ${ }^{1,2}$,

B. Massa ${ }^{1,2}$,

P. Dario ${ }^{1,2}$,

R. Lazzarini ${ }^{1,2}$,

M. Zecca ${ }^{1,2}$,

S. Micera ${ }^{1,2}$, and P. Pastacaldi ${ }^{3}$

${ }^{1}$ Mitech Lab, Scuola Superiore Sant'Anna

via G. Carducci, 40

56127 Pisa, Italy

TEL: +39.050 .883409$

FAX: +39.050 .883402$

E-mail: m.c.carrozza@mail-arts.sssup.it

${ }^{2}$ Centro INAIL RTR

via della Vetraia, 7

55049 Viareggio (LU), Italy

TEL: +39.0584 .385376$

FAX: +39.0584 .385359$

${ }^{3}$ Azienda Ospedaliera Pisana

Presidio Cisanello - via Paradisa n. 2

56100 Pisa, Italy

TEL: +39.050.992111 - +39.050.996111 


\begin{abstract}
Current prosthetic hands are basically simple grippers with one or two degrees of freedom, which barely restore the capability of the thumb-index pinch. Although most amputees consider this performance as acceptable for usual tasks, there is ample room for improvement by exploiting recent progresses in mechatronics design and technology. We are developing a novel prosthetic hand featured by multiple degrees of freedom, tactile sensing capabilities, and distributed control. Our main goal is to pursue an integrated design approach in order to fulfill critical requirements such as cosmetics, controllability, low weight, low energy consumption and noiselessness. This approach can be synthesized by the term “biomechatronic design”, which means developing mechatronic systems inspired by biological world.

This paper describes the first implementation of one single finger of a future biomechatronic hand. The finger has a modular design, which allows to obtain hands with different degrees of freedom and grasping capabilities. Current developments include the implementation of a hand comprising three fingers (opposing thumb, index and middle) and an embedded controller.
\end{abstract}

\title{
1. Introduction
}

The development of an upper limb prosthesis that can be felt as a part of the body by the amputee (Extended Physiological Proprioception - EPP [28]), and that can substitute the amputated limb by closely replicating its sensory-motor capabilities ("cybernetic" prosthesis [8]), is far to become reality. In fact, current commercial prosthetic hands are unable to provide enough grasping functionality 
and to provide sensory-motor information to the user. One of the main problems of the current available devices is the lack of degrees of freedom (DOFs).

Commercially available prosthetic devices, such as Otto Bock SensorHand ${ }^{\mathrm{TM}}$, as well as multifunctional hand designs [1][2][4][9][14][27][31] are far from providing the manipulation capabilities of the human hand [5]. This is due to many different reasons. For example, in prosthetic hands active bending is restricted to two or three joints, which are actuated by a single motor drive acting simultaneously on the metacarpo-phalangeal (MP) joints of the thumb, of the index and of the middle finger, while other joints can bend only passively.

The way to overcome all these problems is to develop a "cybernetic" prosthesis following a biomechatronic approach, i.e. by designing a mechatronic system inspired by the biological world. This goal can be achieved by pursuing two different fundamental objectives:

1. to design an anthropomorphic prosthesis (e.g., by increasing the DOFs of the hand, by mimicking the natural hand kinematics [31], and by developing specific actuators and "skin-like" sensors [7]);

2. to enhance the user-friendliness of the device (e.g., by developing "natural" man-machine interfaces $[6][16][26]$, and suitable signal processing and control strategies [12][19][20]).

The first step towards the former objective is to enhance the dexterity of the hand by increasing its DOFs. As mentioned by several authors [23][27] the main problem encountered in increasing DOFs is related to the limited space available to integrate actuators within the prosthetic hand.

Recent progresses in micro-engineering technologies allow the fabrication of miniature size intelligent actuators, thus encouraging the development of a new 
generation of artificial hands. This is demonstrated by the growing number of publications on this issue appeared in the last few years $[3][14][17][18][24][25][29]$. Innovative micro-actuator technologies such as Shape Memory Alloy (SMA) or ultrasound miniature motors can potentially provide the solution for obtaining more dexterous artificial hands. The introduction of innovative micro-actuators allows to increase the number of active joints, since these actuators can be integrated inside the structure of the prosthetic hand in the palm or even in the fingers. This actuator architecture represents the intrinsic muscular system of the hand, while the extrinsic muscular system [15] can not be replaced, since a prosthetic hand have to suit all amputation levels.

This paper presents preliminary results of a research addressed to the objectives outlined above: to develop an artificial hand with micro-actuators "embedded" on board. The hand will be designed according to a biomechatronic approach, i.e. aiming to integrate micro-mechanisms, micro-actuators, microsensors, processing and controlling micro-electronics, and cosmetic packaging in one artificial hand reproducing as well as possible the performance and the appearance of the natural one.

The design approach, the architecture of the actuators system and the kinematics of the finger transmission are described in this paper. In addition, the first implementation and experimental evaluation of a prosthetic finger incorporating two micro-electromagnetic motors is illustrated. The microactuators were used as linear actuators to directly drive the MP joint and the proximal inter-phalangeal (PIP) joint, while the driving force is transmitted to the distal inter-phalangeal (DIP) joint by using a four-bars linkage. Finally, some 
considerations on the future integration of sensors in the fingers and of the fingers in an artificial hand are presented.

\section{Motivation for a Biomechatronic Approach}

The adoption of bulky and heavy actuators in current limb prostheses led to an extreme reduction of available DOFs. The result of this lack of DOFs is that the fingers are not able to wrap around the object during a general grasping task. Due to this, contact area between the fingers and the grasped object is small, and thus high grip forces are required to perform a stable grasp.

The final consequence on the prosthetic hand design is that a stable grasp can be achieved only by means of large volume actuators, which must be able to supply enough force.

This conventional approach to prosthetic hand design can be represented as the loop described in Fig. 1.

\section{FIG. 1 ABOUT HERE}

The scheme depicted in Fig.1 shows how this approach produces artificial hands with a maximum of two DOFs, and able to provide a pinch force of about 100 N. Artificial hands designed and fabricated with this approach have achieved high quality and reliability, as those produced by Otto Bock Orthopedic Industry Inc. (Duderstadt, DE) or those manufactured by Hosmer Dorrance Corp. (Campbell, CA, USA), but these prostheses still suffers from the same limitations.

The approach proposed in this paper (see Fig. 2) is to invert the loop by using smaller actuators, addressing the objective of increasing DOFs.

FIG. 2 ABOUT HERE 
The consequent enhancement of dexterity and functionality intends to represent the first step to the development of a biomechatronic hand. In particular, to demonstrate the feasibility of this approach we developed a two DOF prosthetic finger actuated by two micro-drivers (based on DC brushless motor) $5 \mathrm{~mm}$ diameter. Due to the consequent enhanced mobility, the novel finger is able to provide an increased contact area between the phalanges and the object during a grasping task. According to our approach, we can accept a reduction in power actuation with the benefit of increasing contact areas and finally of enhancing grip stability.

\section{Design of the biomechatronic hand}

The main requirements to be considered since the very beginning of a prosthetic hand design are the following: cosmetics, controllability, noiselessness, lightness and low energy consumption. These requirements can be fulfilled by implementing an integrated design approach aimed at embedding different functions (mechanisms, actuation, sensing and control) within a housing closely replicating the shape, size and appearance of the human hand. This approach can be synthesized by the term: "biomechatronic" design.

\subsection{Architecture of the Biomechatronic Hand}

The biomechatronic hand will be equipped with three actuator systems to provide a tripod grasping: two identical finger actuator systems and one thumb actuator system.

The finger actuator system is based on two micro-actuators, which drives the MP and the PIP joints respectively; for cosmetic reasons, both actuators are fully integrated in the hand structure: the first in the palm and the second within the 
proximal phalange. The DIP joint is passively driven by a four bars link connected to the PIP joint.

The grasping task is divided in two subsequent phases in which the two different actuator systems are active:

1) reaching and shape adapting phase;

2) grasping phase with thumb opposition.

In fact, in phase one the first actuator system allows the finger to adapt to the morphological characteristics of the grasped object by means of a low output torque motor. In phase two, the thumb actuator system provides a power opposition useful to manage critical grasps, especially in case of heavy or slippery objects.

It is important to point out that the most critical problem of the proposed configuration is related to the strength required to micro-actuators to withstand the high load applied during the grasping phase.

\subsection{Kinematics architecture}

According to the proposed biomechatronic approach, the design goal of our prosthetic hand is to reproduce the kinematics of the natural hand as much as possible (see Fig. 3). Index and middle finger are equipped with two active DOFs respectively in the MP and in the PIP joints, while the PIP joint is actuated by one driven passive DOF.

The thumb is equipped with two active DOFs in the MP joint and one driven passive DOF in the IP joint. This configuration will permit to oppose the thumb to each finger. 
In order to demonstrate the feasibility of the described biomechatronic approach, we started by developing one finger (index or middle).

FIG. 3 ABOUT HERE

\section{Design of the finger prototype}

As outlined above, the two DOF finger prototype is designed by reproducing, as closely as possible, the size and kinematics of a human finger. It consists of three phalanges and of palm housing, which is the part of the palm needed to house the proximal actuator (see Fig. 4).

FIG. 4 ABOUT HERE

\subsection{Actuator system architecture}

In order to match the size of a human finger, two micro-motors are incorporated, respectively, in the palm and in the proximal phalange. This high integration level is achieved by enclosing the motors in a shell housing, where they are constrained only by friction forces. The shell housing is obtained directly from the structure of the proximal phalange.

The actuator system is based on Smoovy ${ }^{\mathrm{TM}}$ (RMB, Eckweg, $\left.\mathrm{CH}\right)$ microdrivers $(5 \mathrm{~mm}$ diameter) high precision linear actuators based on bi-directional DC brushless motors with planetary gears [4] (see Fig. 5). The rotary motion of the shaft is converted to linear motion using lead screw transmission.

FIG. 5 ABOUT HERE

The main mechanical characteristics of the linear actuators declared by manufacturer are listed below (see Table 1).

TABLE 1 ABOUT HERE 
In principle, the selected actuator fulfils almost all the specifications for application in the prosthetic finger: small size, low weight and high bandwidth. The main problem encountered is related to noise, which in present implementation turns out to be too high to be tolerated by prosthesis users. Despite of this limitation, we decided to proceed with the application of the linear actuator in order to investigate integration problems and global performance. One possible solution for reducing noise caused by motors activation is to adjust the acoustical impedance of the motors housing and of the external palm/finger structure.

The output force resulting from motor activation is sufficient to move the phalanges for achieving adaptive grasp. In addition, the shell housing provides mechanical resistance of the shaft to both axial and radial loads. This turns out to be essential during grasping tasks, where loads, derived from the thumb opposition, act both on the actuator system and on the whole finger structure.

\subsection{Kinematics architecture}

The kinematics of each finger joint is described in detail in the following subsections.

\subsubsection{MP Joint}

The proximal actuator is integrated in the palm and transmits the mechanical power through a slider crank mechanism to the proximal phalange providing flexion/extension movement (see Fig. 6). The slider is driven by the lead screw transmission directly mounted on the motor shaft. Member 1 is the connecting linkage and member 2 represents the proximal phalange. 
Geometrical relations of the slider crank mechanism are:

$$
\left\{\begin{array}{l}
d_{1} \sin \alpha+c=d_{2} \sin \vartheta \Rightarrow \alpha=\alpha(\vartheta) \\
d-x=d_{1} \cos \alpha-d_{2} \cos \vartheta \Rightarrow \vartheta=\vartheta(x)
\end{array}\right.
$$

Symbols $c, d, d_{1}, d_{2}, \alpha, \vartheta$, and $x$ refer to geometrical features of the slider crank mechanism and are defined according to Fig. 6.

In order to obtain flexion velocity $\dot{\vartheta}$ of the proximal phalange as a function of translation velocity of the slider $\dot{x}$ we can write:

$$
\begin{aligned}
& \underline{v}_{P}=\dot{x} \underline{i} \\
& \underline{v}_{P}=\underline{v}_{O}+\underline{\omega}_{1} \wedge O P=\underline{v}_{O}+\dot{\alpha} \underline{k} \wedge O P \\
& \underline{v}_{O}=\underline{\omega}_{2} \wedge O^{\prime} O=\dot{\vartheta} \underline{k} \wedge O^{\prime} O \\
& \dot{x} \underline{i}=\dot{\vartheta} \underline{k} \wedge O^{\prime} O+\dot{\alpha} \underline{k} \wedge O P=-d_{2} \dot{\vartheta} \sin \vartheta \underline{i}+d_{2} \dot{\vartheta} \cos \vartheta \underline{j}+ \\
&+d_{1} \dot{\alpha} \sin \alpha \underline{i}-d_{1} \dot{\alpha} \cos \alpha \underline{j}
\end{aligned}
$$

where $\underline{\mathrm{i}}, \underline{\mathrm{j}}$ and $\underline{\mathrm{k}}$ are the three orthogonal versors, $\underline{\mathrm{v}}_{\mathrm{O}}$ and $\underline{\mathrm{v}}_{\mathrm{P}}$ are the velocities of points $\mathrm{O}$ and $\mathrm{P}$, respectively and $\underline{\omega}_{1}$ and $\underline{\omega}_{2}$ are the angular velocities of the OP and of the OO' link, respectively.

Projecting along the horizontal and vertical axes (described by versors $\underline{i}$ and $\underline{\mathrm{j}}$ ), we obtain:

$$
\left\{\begin{array}{l}
\dot{\mathrm{x}}=-\mathrm{d}_{2} \dot{\vartheta} \sin \vartheta+\mathrm{d}_{1} \dot{\alpha} \sin \alpha \\
0=\mathrm{d}_{2} \dot{\vartheta} \cos \vartheta-\mathrm{d}_{1} \dot{\alpha} \cos \alpha
\end{array}\right.
$$

Substituting the second equation in the first equation we find the solution:

$$
\begin{aligned}
& \dot{\alpha}=\frac{d_{2}}{d_{1}} \frac{\cos \vartheta}{\cos \alpha} \dot{\vartheta} \\
& \dot{x}=d_{2}(\cos \vartheta \operatorname{tg} \alpha-\sin \vartheta) \dot{\vartheta} \Rightarrow \dot{\vartheta}=\dot{\vartheta}(\vartheta, \dot{x})
\end{aligned}
$$


In Fig. 7, the function $\dot{\vartheta}=\dot{\vartheta}(\vartheta, \dot{x})$ for the MP joint is showed. Where $\dot{x}$ is the maximum linear velocity of the micro-actuators $(200[\mathrm{~mm} / \mathrm{min}]), \dot{\vartheta}$ is the MP angular velocity and $\vartheta$ the MP angular position (30 [deg]: full extension and 120 [deg]: full flexion).

FIG. 7 ABOUT HERE

\subsubsection{PIP joint}

The same mechanism used for the MP moves the PIP joint. Only the geometrical features are varied (see Table 2) in order to fit within the space available according to the specifications of the biomechatronic hand.

TABLE 2 ABOUT HERE

High friction forces occur, because of mechanism movement, during the low pitch of the threaded shafts. For this reason the two lead screw transmissions are non backdrivable; but this turns out to be useful for ensuring grasping forces maintenance without power supplying.

\subsubsection{DIP joint}

A four bars link has been adopted for the DIP joint and its geometrical features have been designed in order to reproduce as closely as possible natural DIP joint flexion. According to the three prescribed positions method [11] we synthesized the mechanism, where length of the links A-D is showed in Table 3.

\section{TABLE 3 ABOUT HERE}

The selected positions were the extended position, the flexed position and the intermediate position of the DIP joint, according to position assumed by the natural finger. These positions are illustrated in Table 4. 


\section{TABLE 4 ABOUT HERE}

\section{Fabrication of the finger prototype}

A first prototype of the finger was fabricated using the Fused Deposition Modeling [FDM] process (see Fig. 8). This process allows the fabrication in a single process of three-dimensional objects, made out of acrylonitrile/butadiene/styrene $[\mathrm{ABS}]$ resin, directly from CAD-generated solid models. This rapid prototyping technique allows to make devices in order to make preliminary tests of different design solutions without the cost and time constraints typical of traditional prototyping technologies.

FIG. 8 ABOUT HERE

\section{Fingertip Force Characterization}

A first set of experimental tests has been performed in order to evaluate the force that the finger is able to exert on an external object [19]. To this aim we have measured the force resulting when the finger is pressing directly on a force sensor, corresponding to different configurations of the joints.

The finger prototype was mounted on a four DOF manipulator (X, Y, Z translation plus one DOF for tilting) as depicted in Fig. 9a and 9b. The force sensor was a 3-axial piezoelectric load cell (9251 A, Piezo-Instrumentation KISTLER, Kiwag, $\mathrm{CH}$ ); the sensor was mounted on a steel plate and covered by an aluminum plate in order to provide to the finger a contact area to apply the force. The load cell was connected to charge amplifier (Piezo-Instrumentation KISTLER, Kiwag, CH); the analog signal was converted by a digital oscilloscope (TDS 220, Tektronix, Beaverton, US) and acquired through a PC (see Fig. 9a 
and 9b) using WaveStar (Tektronix). Each Smoovy ${ }^{\mathrm{TM}}$ actuator is controlled by a CCS00001 controller (RMB).

\section{FIG. 9a AND 9b ABOUT HERE}

The finger position was adjusted in order to obtain an exerted force parallel to the Z-axis of the load cell. Two "pressing" tasks were identified in order to evaluate separately and independently force obtained by the two actuators incorporated in the finger:

- TASK 1: the pushing action was exerted only by the distal actuator;

- TASK 2: the pushing action was exerted only by the proximal actuator.

Corresponding to each task, two subtasks were identified according to the position of the non-active joint (extended, flexed). The different values of joint rotation angles corresponding to each subtask are illustrated in Table 5 and Fig. 10.

\subsection{Experimental Set-up}

During the force characterization the fingertip pushed on the force sensor. The $\mathrm{Z}$ force component was recorded, the $\mathrm{X}$ and $\mathrm{Y}$ outputs of the load cell were monitored and led to zero. This was obtained by adjusting the finger position for obtaining a force parallel to the Z-axis of the load cell. A first set of experimental tests was done on the finger prototype, with the aim of evaluating how much force the finger is able to apply on an object.

\section{TABLE 5 ABOUT HERE}

FIG. 10 ABOUT HERE

\subsection{Results and discussion}

Ten tests were performed for each subtask. The obtained results are summarized in Table 6 and illustrated in Fig. 11. Table 6 also reports the expected values 
(without taking into account power losses) of the fingertip force, according to the calculations previously illustrated (see Section 4.2). These force values are comparable with force exerted by "natural" human finger during fine manipulation, thus demonstrating the feasibility of the biomechatronic approach, at least for this class of manipulation tasks [22].

We noticed a higher discrepancy between theoretical and measured force values during the different trials implementing Task 1 (more than 1,000 $\mathrm{mN}$ (theoretical) versus about $600 \mathrm{mN}$ (experimental)) than during Task $2(1,141 \mathrm{mN}$ versus $990 \mathrm{mN}$ ). These differences are possibly related to the friction forces acting during the movement of the finger; in particular during Task 1 these losses are greater because of the action of the 4-bars link driving the DIP joint.

It is important to point out that all the values showed a quite narrow standard deviation (less than 3.3\%) among each set, proving a good repeatability of the force developed by the biomechatronic finger.

These force levels are sufficient to accomplish the first phase of the grasping task (reaching and shape adapting). For the second phase (grasping with thumb opposition) we are developing the thumb actuator system, based on DC motor, able to provide sufficient grasping forces.

TABLE 6 ABOUT HERE

FIG. 11 ABOUT HERE

\section{Future improvements}

The experimental tests showed promising results, but there is still room for improvement. First of all, natural fingers movements during grasping activities 
will be further investigated in order to achieve a truly "human-like" behaviour of the prosthetic finger.

A micro-sensory system, incorporating multi-component force sensors and joint position sensors, will be integrated in the mechanical structure of the finger in order to sense incipient slippage and force sensing abilities. Finally, suitable control strategies will be investigated and applied in order to develop a smart and user friendly control interface for the prosthetic hand.

\section{Conclusions}

A novel approach to the design and fabrication of innovative prosthetic hands, called biomechatronic approach, has been presented. It is based on integrating together multiple degrees of freedom, multi-sensing capabilities, and distributed control in order to obtain "graceful" human-like appearance, simple and direct controllability, low weight, low energy consumption and noiselessness of the prosthetic hand.

Following this type of approach a first prototype of an active finger with two DOFs has been designed and fabricated.

In this paper we focused our attention on the innovative integration of micro-electromagnetic actuators within the finger structure as the first step to develop a biomechatronic prosthetic hand.

\section{Acknowledgements}

This work has been supported by a research project entitled "Design and development of innovative components for sensorized prosthetic systems" currently ongoing at the "Applied Research Center on Rehabilitation Engineering" funded by INAIL (National Institute for Insurance of Injured 
Workers), and originated by a joint initiative promoted by INAIL and by Scuola Superiore Sant'Anna.

The authors are also grateful to Mr. Carlo Filippeschi and Mr. Gabriele Favati for their valuable technical assistance. The authors would also thank Mr. Rinaldo Sacchetti for helpful discussions and criticism on the biomechatronic prosthetic hand concept.

\section{References}

[1] P.J. Agnew, Functional effectiveness of a myoelectric prosthesis compared with a functional split hook prosthesis: a single subject experiment, Prost. \& Orth. Int. 5 (1981), 92-96.

[2] S.-E. Baek, S.-H. Lee, J.-H. Chang, Design and control of a robotic finger for prosthetic hands, Proc. Int. Conf .Intelligent Robots and Systems (1999), $113-117$.

[3] J. Butterfass, G. Hirzinger, S. Knoch, H. Liu, DLR's multy sensory articulated hand Part I: hard and software architecture, Proc. Int. Conf. Robotics \& Automation (1998), 2081-2086.

[4] M. E. Cupo, S. J. Sheredos, Clinical Evaluation of a new, above elbow, body powered prosthetic arm: a final report, J. Rehab. Res. Dev. 35 (1998), $431-446$.

[5] M. R. Cutkosky, Robotic Grasping and Fine Manipulation, Boston: Kluwer Academic Publishers, 1985.

[6] P. Dario, P. Garzella, M. Toro, S. Micera, M. Alavi, U. Meyer, E. Valderrama, L. Sebastiani, B. Ghelarducci, C. Mazzoni, P. Pastacaldi, 
Neural Interfaces for Regenerated Nerve Stimulation and Recording, IEEE Trans. Rehab. Eng. 6 (1998), 353-363.

[7] P. Dario, R. Lazzarini, R. Magni, An Integrated Miniature Fingertip Sensor, Proc. VII Int. Symp. on Micro Machine and Human Science (MHS'96) (1996), 91-97.

[8] J. A. Doeringer, N. Hogan, Performance of above elbow body-powered prostheses in visually guided unconstrained motion task, IEEE Trans. Rehab. Eng. 42 (1995), 621-631.

[9] R. Doshi, C. Yeh, M. LeBlanc, The design and development of a gloveless endoskeletal prosthetic hand, J. Rehab. Res. Dev. 35 (1998), 388-395.

[10] A. Drapp, Smoovy ${ }^{\mathrm{TM}}$ : a new modular approach for miniaturization, Proc. Int. Conf. Actuator (1998), 566-567.

[11] A. G. Erdman, G. N. Sandor, Mechanism design. Prentice Hall International, Inc. Third edition (1997).

[12] C. Freschi, A. Di Giglio, S. Micera, A. M. Sabatini and P. Dario, Hybrid Control of Sensorised Hand Prosthesis: Preliminary Work, EUREL Conf. (1999).

[13] I. A. Kapandji, The physiology of the joints, Churchill Livingstone, Volume I, Fifth edition, 1982.

[14] P. J. Kyberd, O. E. Holland, P. H. Chappel, S. Smith, R. Tregidgo, P. J. Bagwell, and M. Snaith, MARCUS: a two degree of freedom hand prosthesis with hierarchical grip control, IEEE Trans. Rehab. Eng. 3 (1995), 70-6.

[15] I. A. Kapandji, The physiology of the joints, Churchill Livingstone, Volume I, Fifth edition, 1982. 
[16] G. T. A. Kovacs, J. M. Rosen, Regeneration-Type Peripheral Nerve Interfaces for Direct Man/Machine Interface, in Robots and Biological Systems: Towards a New Bionics?, P. Dario, G. Sandini, and P: Aebischer, Eds., NATO ASI Series F. 102 (1993).

[17] Y. K. Lee, I. Shimoyama, A skeletal framework artificial hand actuated by pneumatic artificial muscles, Proc. Int. Conf. Robotics \& Automation (1999), 926-931.

[18] C. S. Lovchik, M. A. Diftler, The robonaut hand: a dextrous robot hand for space, Proc. Int. Conf. Robotics \& Automation (1999), 907-912.

[19] S. Micera, A.M. Sabatini, P. Dario, B. Rossi, A Hybrid Approach for EMG Pattern Analyis for Classification of Arm Movements, Med Eng Phys 21 (1999), 303-311.

[20] A. Mingrino, A. Bucci, R. Magni, P. Dario, Slippage Control in Hand Prostheses by Sensing Grasping Forces and Sliding Motion, Proc. of IEEE/RSJ/GI Int. Conf. Intelligent Robots and Systems, (1994), 1803-1809.

[21] K. Nagai, Y. Eto, D. Asai, M. Yazaki, Development of a three-fingered robotic hand-wrist for compliant motion, Proc. Int. Conf. Intelligent Robots and Systems (1998), 476-481.

[22] D. T. V. Pawlock, R. D. Howe, Dynamic contact of the human fingerpad against a flat surface, ASME J Biomech Eng 121 (1999), 605-611.

[23] J. L. Pons, R. Ceres, F. Pfeiffer, Multifingered dextrous robotics hand design and control: a review, Robotica 17 (1999), 661-674.

[24] J. L. Pons, H. Rodriguez, R. Ceres, Reynaerts, W. V. Moorleghem, Study of SMA actuation to develop a modular, user-adaptable hand prosthesis, Proc. Int. Conf. Actuator (1998), 490-493. 
[25] T. Raparelli, G. Mattiazzo, S. Mauro, M. Velardocchia, Design and development of a pneumatic anthropomorphic hand, J Robotic Systems 17 (2000), 1-15.

[26] R. R. Riso, Strategies for providing upper extremity amputees with tactile and hand position feedback - moving closer to the bionic arm, Technol Health Care 7:6 (1999), 401-409.

[27] D. H. Silcox, M. D. Rooks, R. R. Vogel, L. L. Fleming, Myoelectric Prostheses, J. Bone \& Joint Surg., 75 (1993), 1781-1789.

[28] D. C. Simpson, The Choice of Control System for multimovement prostheses: Extended Physiological Proprioception (EPP), in The Control of Upper-Extremity Prostheses and Orthoses, P. Herberts et al., Eds., 1974.

[29] Special Session on Actuators for Artificial Limbs, ACTUATOR 2000, Bremen, Germany (2000).

[30] S. T. Ventakaraman, T. Iberall, Dextrous robot hands. Panel discussion. Springer-Verlag, 1989, 287-298.

[31] R. Vinet, Y. Lozac'h, N. Beaundry, G. Drouin, Design methodology for a multifunctional hand prosthesis, J. Rehab. Res. Dev. 32 (1995), 316-324. 


\begin{tabular}{|l|l|}
\hline Gear stages & 3 \\
\hline Transmission rate & $1: 125$ \\
\hline Maximum load radial & $25 \mathrm{~N}$ \\
\hline Maximum load axial & $40 \mathrm{~N}$ \\
\hline Maximum speed & $200 \mathrm{~mm} / \mathrm{min}$ \\
\hline Nominal force & $12 \mathrm{~N}$ \\
\hline Weight & $3.2 \mathrm{~g}$ \\
\hline
\end{tabular}

Table 1: Summary of the main characteristics of the Smoovy ${ }^{\mathrm{TM}}$ (RMB, Eckweg, $\mathrm{CH})$ micro drivers (5 $\mathrm{mm}$ diameter). 


\begin{tabular}{|l|l|l|}
\hline & PIP joint & MP joint \\
\hline d1 & $9 \mathrm{~mm}$ & $18 \mathrm{~mm}$ \\
\hline d2 & $4 \mathrm{~mm}$ & $6 \mathrm{~mm}$ \\
\hline C & $5 \mathrm{~mm}$ & $6 \mathrm{~mm}$ \\
\hline
\end{tabular}

Table 2: Geometrical features of the slider crank mechanism of the MP and of the PIP joints. 


\begin{tabular}{|l|l|}
\hline A & $5.2 \mathrm{~mm}$ \\
\hline B & $28.7 \mathrm{~mm}$ \\
\hline C & $3.6 \mathrm{~mm}$ \\
\hline D & $25.1 \mathrm{~mm}$ \\
\hline
\end{tabular}

Table 3: Geometrical features of the four bars link mechanism (see also Fig. 6 and Fig. 8). 


\begin{tabular}{|l|l|l|}
\hline$\alpha=180$ & $\beta=180$ & Full extension \\
\hline$\alpha=150$ & $\beta=168.5$ & Intermidiate position \\
\hline$\alpha=100$ & $\beta=102$ & Full flection \\
\hline
\end{tabular}

Table 4: Prescribed positions (see also Fig. 6) for four bars linkage synthesis. 


\begin{tabular}{|l|c|c|c|c|}
\hline & \multicolumn{2}{|c|}{ TASK 1 } & \multicolumn{2}{c|}{ TASK 2 } \\
\hline Position & MP Joint [deg] & PIP Joint [deg] & MP Joint [deg] & PIP Joint [deg] \\
\hline 1 & 0 & 60 & 60 & 0 \\
\hline 2 & 30 & 45 & 30 & 45 \\
\hline
\end{tabular}

Table 5: Pressing positions (see also Fig. 11). 


\begin{tabular}{|l|l|l|l|l|}
\hline & Task 1_1 & Task 1_2 & Task 2_1 & Task 2_2 \\
\hline Mean Force $(\mathrm{mN})$ & 586 & 624 & 848 & 990 \\
\hline Standard Deviation $(\%)$ & 2,84 & 3,29 & 2,00 & 2,07 \\
\hline Expected Value $(\mathrm{mN})$ & 1057 & 1059 & 951 & 1143 \\
\hline
\end{tabular}

Table 6: Mean values and standard deviation of force exerted by the finger prototype during test run in different tasks. Tasks correspond to specific joint positions as defined in Table 5 . 


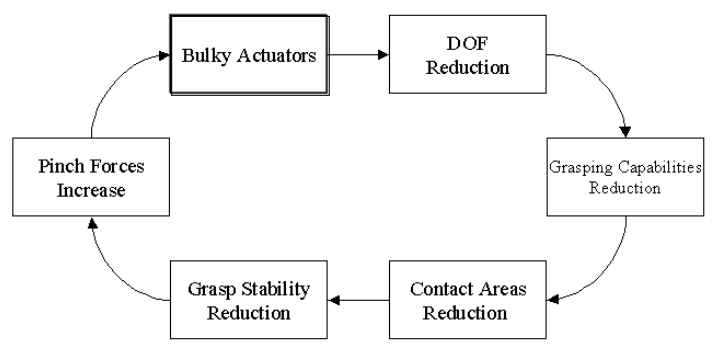

Fig. 1: Loop corresponding to conventional approach to prosthetic hand design. 


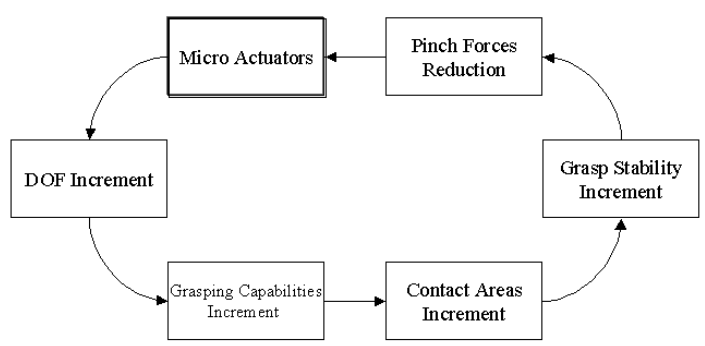

Fig. 2: Loop corresponding to the biomechatronic approach to prosthetic hand design. 


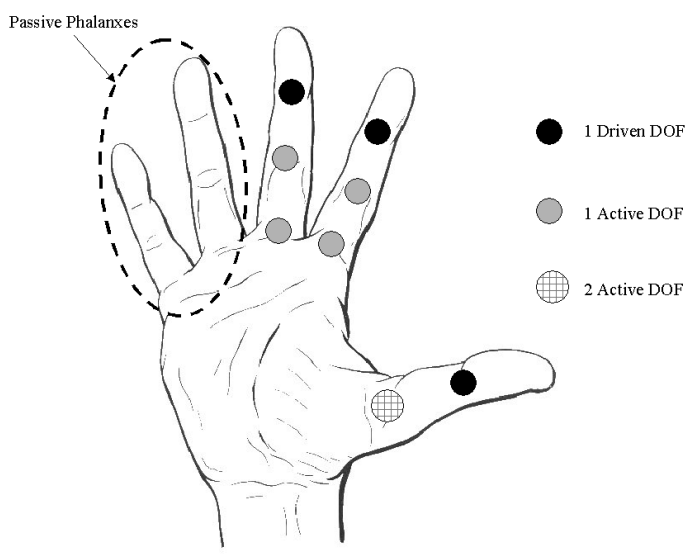

Fig. 3: Kinematic architecture of the "natural" hand 


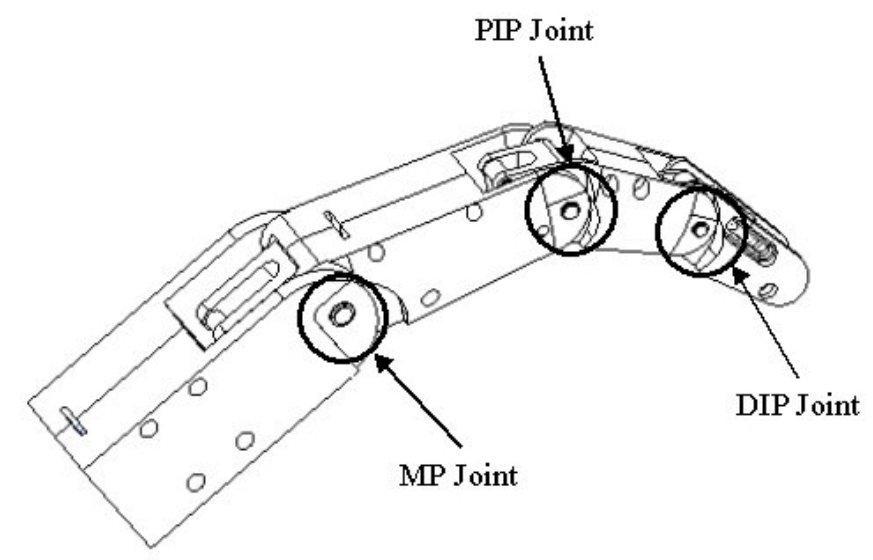

Fig. 4: General assembling of the finger prototype. 


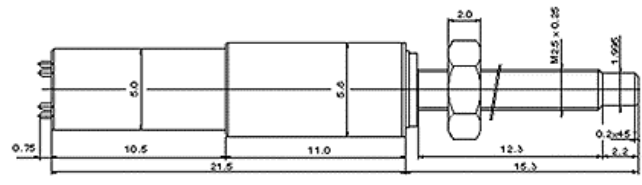

Fig. 5: Longitudinal drawing of the Smoovy (RMB) linear microactuator. 


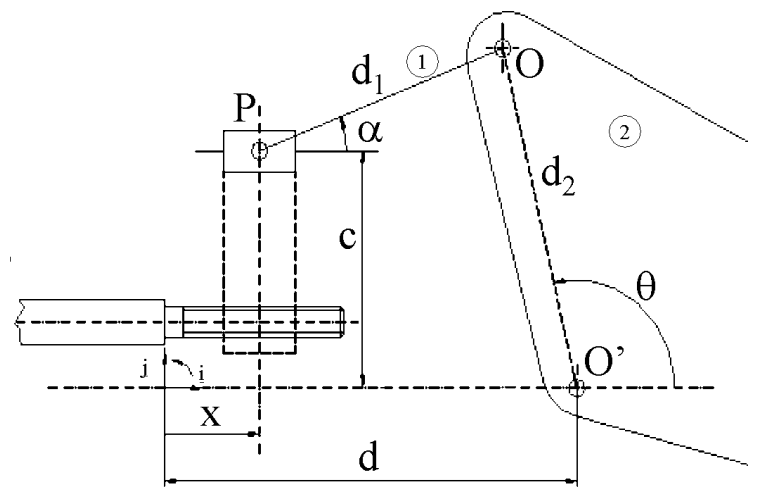

Fig. 6: Detailed drawing of the crank slider mechanism in the MP joint 


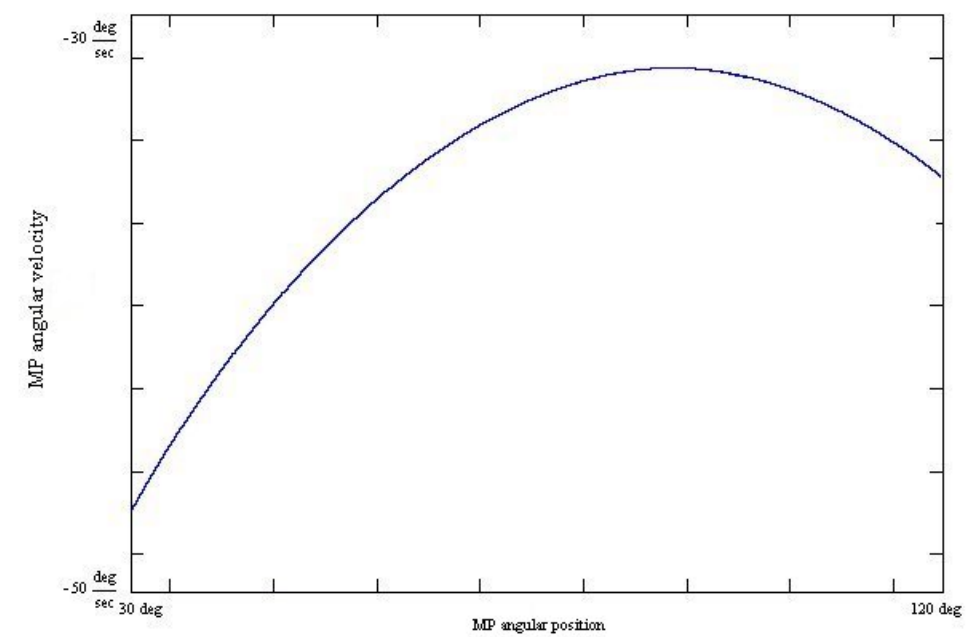

Fig. 7: MP angular velocity $\dot{\theta}$ vs. MP angular position $\theta$ expected from calculations (see Fig. 6 for variable definitions). 


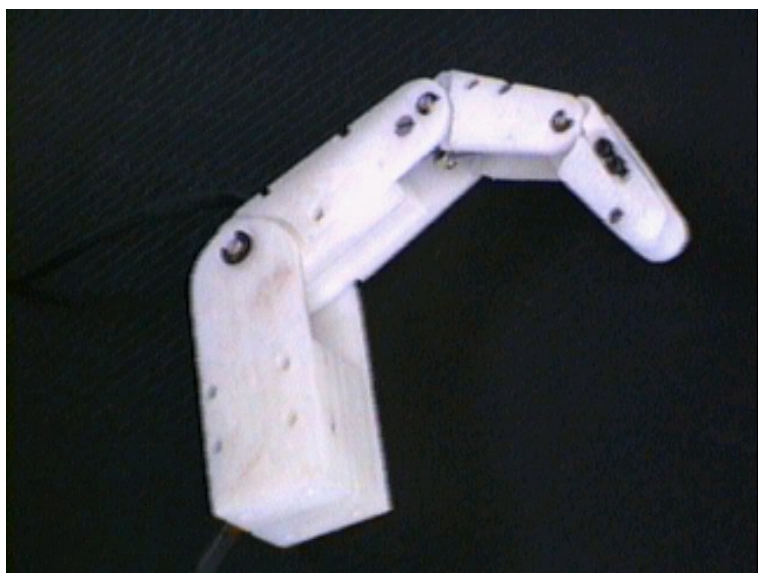

Fig. 8: Photograph of the finger prototype. 


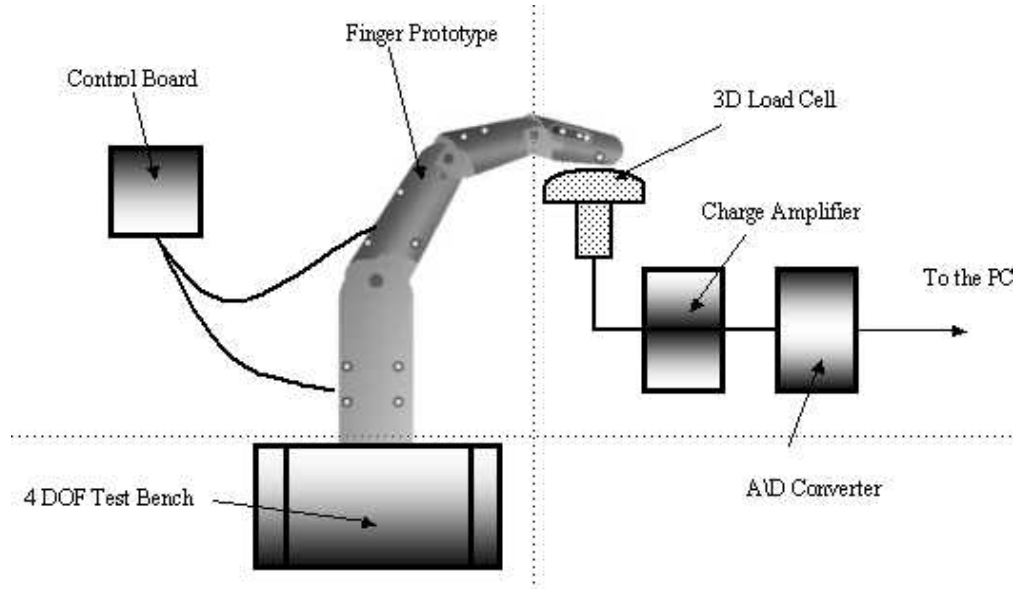

Fig. 9a: Schematic drawing of the experimental set-up. 


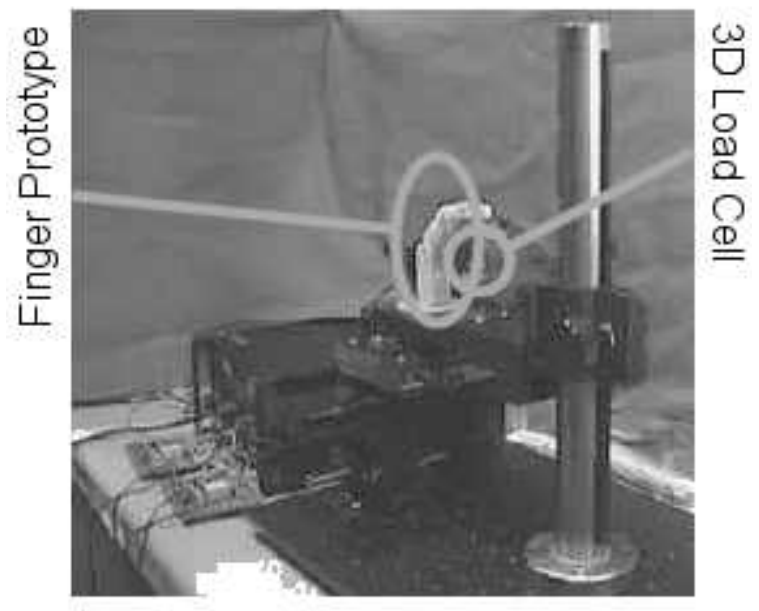

Fig. 9b: Photograph of the experimental set-up. 


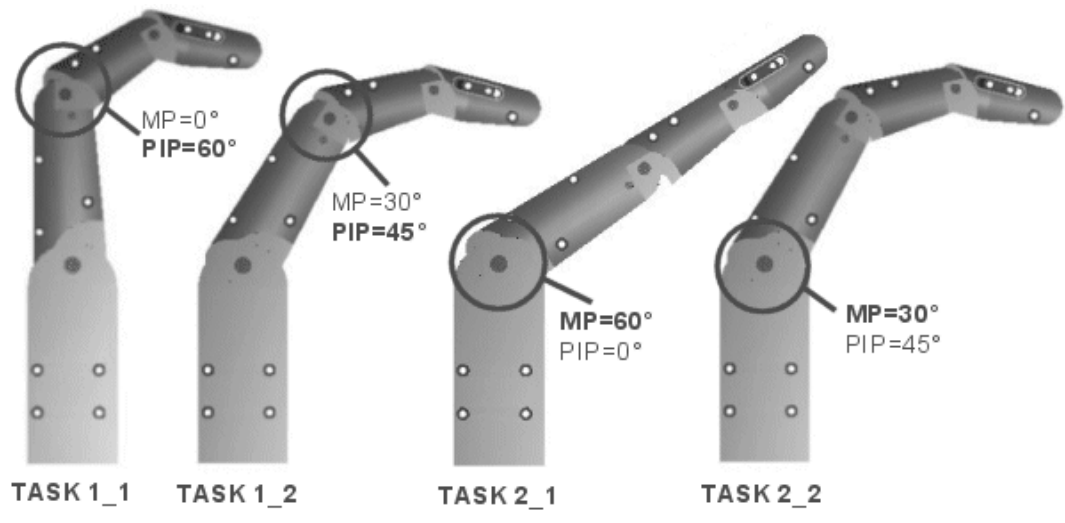

Fig. 10: Different positions of finger joints for each task. The active joint for each task and position is indicated by a small circle. 


\section{Force Characterization}

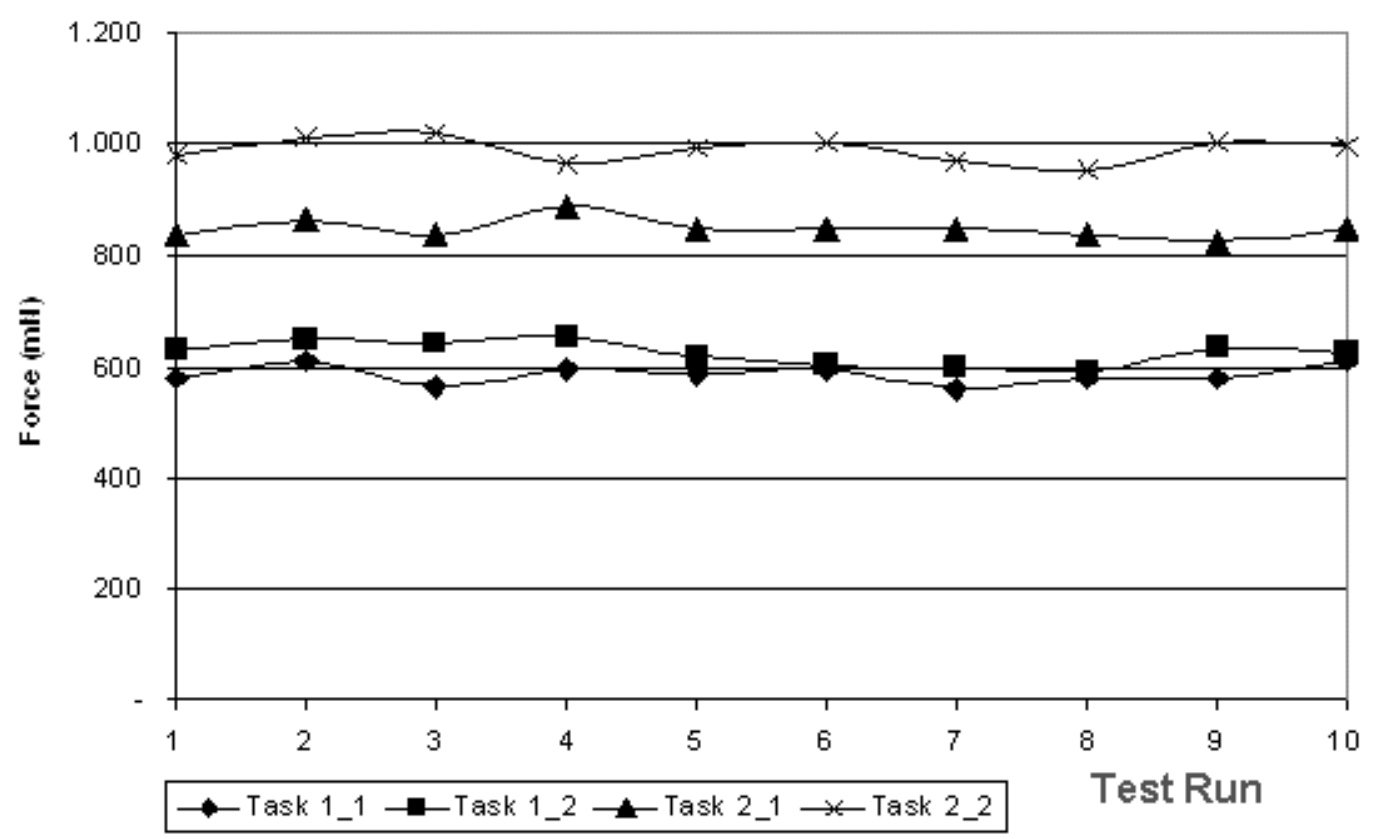

Fig. 11: Experimental results. The number of the task is chosen according to Table 5. 